\title{
Radiosensitivity in Escherichia coli
}

\author{
By J. GREENBERG AND PEARL WOODY-KARRER \\ Palo Alto Medical Research Foundation, 860 Bryant Street, \\ Palo Alto, California, U.S.A.
}

(Received 29 April 1963)

\begin{abstract}
SUMMARY
A radiosensitive mutant of Escherichia coli strain K12, w3747 was found to be similar to $E$. coli strains B and s with respect to survival following ultraviolet irradiation, plating-medium recovery, ability to 'reactivate' irradiated $\mathrm{T} 1$ phage, and in its cross-resistance pattern with radiomimetic chemicals. In all these respects the radiosensitive $\boldsymbol{E}$. coli strain $\mathrm{k} \mathbf{1 2}$, $A B 1186$, isolated by Howard-Flanders, was found to resemble the Hill radiation hypersensitive strain $B_{8-1}$, and, except in the ability to reactivate irradiated $T 1$ phage, strain $B_{8-2}$. Strains $w_{3747_{s}}$ and $A B 1886$ acquired sensitivity to radiomimetic chemicals concomitantly with radiation sensitivity. Strains $\mathrm{B}_{\mathrm{g}-1}$ and $\mathrm{B}_{\mathrm{g}-2}$ on becoming hypersensitive to radiation did not become correspondingly hypersensitive to radiomimetic chemicals. In fact, they acquired a small degree of resistance to these chemicals, relative to strain $\mathrm{B}$, and a substantial increase in resistance to 1-methyl-3nitro-1-nitrosoguanidine and proflavine. These results are discussed in terms of known genetic loci implicated in radiation sensitivity and resistance.
\end{abstract}

\section{INTRODUCTION}

Escherichia coli strains B and s are naturally sensitive to ultraviolet (u.v.) radiation and X-radiation but sensitivity can be demonstrated only when they are plated, following irradiation, on complex media (Roberts \& Aldous, 1949; Alper \& Gillies, 1960; Hill \& Simson, 1961; Woody, Mandell \& Greenberg, 1961). Many different radiation-resistant mutants of $\boldsymbol{E}$. coli strains B and s have been isolated (Witkin, 1947; Alper \& Gillies, 1960; Greenberg, Mandell \& Woody, 1961 $a$ ) and these exhibit concomitant resistance to a variety of chemical agents such as nitrogen mustard (Bryson, 1948; Woody et al. 1961), nitrofurazone (Szybalski \& Nelson, 1954; Hill \& Simson, 1961; Woody-Karrer \& Greenberg, 1963), Mitomycin C (Greenberg, Mandell \& Woody, 1961b), nitrosoguanidines (Mandell, Woody \& Greenberg, 1961), azaserine (Greenberg et al. 1961 a). These radioresistant mutants have been differentiated on the basis of their cross-resistance patterns with radiomimetic agents. Mutants of $E$. coli strain в have also been isolated which are even more sensitive to radiation than is $E$. coli strain в itself. These include $E$. coli strain $B_{8-1}$ (Hill, 1958), strain $B_{8-2}$ (Hill \& Simson, 1961) and strain $B_{I I I}$ (Rörsch, Edelman, van der Kamp \& Cohen, 1962). Unlike $E$. coli strain ${ }_{B}$, strains $B_{8-1}$ and $B_{8-2}$ do not form long filaments when treated with u.v. radiation. Furthermore, strains $\mathbf{B}_{\mathrm{s}-1}$ and $\mathrm{B}_{\mathrm{III}}$ exhibit a peculiar property when used as hosts for u.v.-irradiated coliphage T1: when irradiated $\mathrm{T} 1$ phage is plated on $E$. coli strains $\mathrm{B}_{\mathrm{B}-1}$ and $\mathrm{B}_{\mathrm{III}}$, it is significantly more sensitive to the lethal effects of irradiation than when it is 
plated on parent $E$. coli strain в. This has been taken to mean that $\boldsymbol{E}$. coli strains $\mathrm{B}_{\mathrm{8}-1}$ and $\mathrm{B}_{\mathrm{III}}$ lack a mechanism, present in other strains of $E$. coli, which repairs the damage caused by u.v. radiation to $\mathrm{T} 1$ phage. On the other hand, E. coli strain $\mathbf{B}_{8-2}$, like its parent, strain $\mathrm{B}$, 'reactivates' irradiated T1 phage.

It would be of considerable significance if genetically controlled variations in radiosensitivity were demonstrable in Escherichia coli strain $\mathrm{k12}$, if only because more is known about strain $\mathrm{k12}$ than about strain $\mathrm{B}$ as a genetic tool. Most derivatives of $E$. coli strain $\mathrm{k} 12$ are radioresistant (Adler \& Copeland, 1962; HowardFlanders \& Theriot, 1962) but strain k12 AB531 was reported by Adler \& Copeland (1962) to be relatively less resistant than other K12 strains tested in their laboratory. However, the ultraviolet dose, about $600 \mathrm{ergs} / \mathrm{mm} .^{2}$, reported to give one decade of kill in strain AB531 is in the same range as that reported for the most frequently occurring radioresistant mutants of $E$. coli strain s (Greenberg \& Woody-Karrer, 1963). Howard-Flanders \& Theriot (1962) isolated a mutant of strain K12 AB1157 (designated AB1886 by Howard-Flanders, Boyce, Simson \& Theriot, 1962), which was very sensitive to u.v. radiation and which lacked the 'reactivating' mechanism for irradiated T1. Another radiation-sensitive mutant of strain k12 was isolated in our laboratory following treatment of strain $\mathrm{K}_{2} \mathrm{~F}_{13}^{\prime}$ (w3747, Hirota \& Sneath, 1961) with acridine orange (Cook \& Greenberg, unpublished observations). This

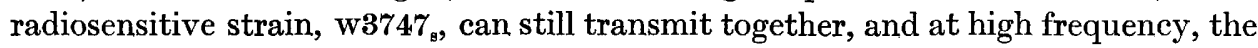
$F$ factor and the ability to ferment lactose. A further description of this mutant was one of the purposes of the present work.

Since increased resistance to radiomimetic chemicals is an invariable concomitant of a mutation to radioresistance in Escherichia coli strains B and s (Greenberg \& Woody-Karrer, 1963), another purpose of these experiments was to see whether mutations from resistance to sensitivity in $E$. coli strain $\mathrm{K} 12$ and from sensitivity to hypersensitivity in strain в were accompanied by a decrease in resistance to radiomimetic agents. Strains $\mathrm{B}, \mathrm{B} / \mathrm{r}, \mathrm{B}_{\mathrm{s}-1}, \mathrm{~B}_{\mathrm{8}-2}, \mathrm{AB} 1886$, $\mathrm{AB} 1157$, w3747 and $\mathrm{w} 3747_{\mathrm{g}}$ were compared for the following properties: ultraviolet survival curves, plating-medium responses, 'reactivation' of irradiated TI phage and cross-resistance patterns with radiomimetic chemicals. The results will show that in all these properties strains $A B 1157$ and $w 3747$ resembled strain $B / r$; strain $A B 1886$ resembled strain $B_{8-1}$ (but not $\mathrm{B}_{\mathrm{B}-2}$ ); and strain $\mathrm{w} 3747_{\mathrm{B}}$ resembled strain $\mathrm{B}$. There are, therefore, two kinds of mutation to radiosensitivity in $E$. coli strain k12. Both of these, significantly, are accompanied by increased sensitivity to radiomimetic chemicals, but the hyperradiosensitive mutants of $E$. coli strain $\mathrm{B}_{\left(\mathrm{B}_{\mathrm{B}-1}, \mathrm{~B}_{\mathrm{B}-2}\right)}$ ) show a decreased sensitivity to most of the radiomimetic chemicals.

\section{METHODS}

Strains of Escherichia coli $u$ sed. The $E$. coli strains used, their derivation, relative radiosensitivities assayed on tryptone agar, and source are listed in Table 1.

Chemical compounds used. The chemicals used were: 1-methyl-3-nitro-1-nitrosoguanidine (which will be referred to as nitrosoguanidine, bought from the Aldrich Chemical Co., Milwaukee, Wisconsin, U.S.A., and recrystallized from ethanol); proflavine hydrochloride (obtained from the Allied Chemical and Dye Corp., New York, N.Y.); nitrogen mustard (a gift from Merck Sharp and Dohme, Rahway, 
N.J.); Mitomycin C (supplied by the Cancer Chemotherapy National Service Centre, Bethesda, Maryland, U.S.A.); nitrofurazone (5-nitro-2-furaldehyde semicarbazone; a gift from Eaton Laboratories, Division of the Norwich Pharmacal Company, Norwich, N.Y.). All compounds were prepared in sterile distilled water immediately before use.

Table 1. Summary of strains

\begin{tabular}{|c|c|c|c|}
\hline $\begin{array}{l}\text { Strain of } \\
E . \text { coli }\end{array}$ & Derivation & $\begin{array}{c}\text { Relative } \\
\text { radiosensitivity } \\
\text { on tryptone agar }\end{array}$ & Source \\
\hline $\mathbf{B}$ & & Sensitive & Dr R. Hill \\
\hline $\mathbf{B}_{8-1}$ & Strain B & Hypersensitive & Dr R. Hill \\
\hline $\mathbf{B}_{8-2}$ & Strain B & Hypersensitive & Dr R. Hill \\
\hline $\mathrm{B} / \mathbf{r}\left(\mathrm{CsH}^{*}\right)$ & Strain B & Resistant & Dr H. I. Adler \\
\hline s & Strain B & Sensitive & Dr A. D. Hershey \\
\hline $\mathrm{s} / \mathrm{MC} \mathrm{Ib}\left(\mathrm{R}_{\mathbf{4}}\right)$ & Strain $\mathbf{s}$ & Resistant & \\
\hline AB1157 & Strain K12 & Resistant & Dr E. Adelberg \\
\hline AB 1886 & Strain AB1157 & Sensitive & Dr P. Howard-Flanders \\
\hline w3747 & Strain $\mathbf{k} 12$ & Resistant & Dr J. Lederberg \\
\hline w3747 & Strain w3747 & Sensitive & \\
\hline
\end{tabular}

Culture media. The media used contained per litre of distilled water :

(1) Tryptone agar: tryptone, $10.0 \mathrm{~g}$.; glucose, $1.0 \mathrm{~g}$.; sodium citrate, $2.0 \mathrm{~g}$.; sodium chloride, 8.0 g.; agar, 12.0 g. (BBL, Baltimore Biological Lab., Inc.); adjusted to $\mathrm{pH} 7 \cdot 0$ or 7.8 with sodium hydroxide or to $\mathrm{pH} 5.5$ with hydrochloric acid.

(2) Tryptone semi-solid agar: tryptone, glucose, sodium chloride, and sodium citrate at the same concentrations as in tryptone agar; agar, 6.0 g.; adjusted to $\mathrm{pH} 7 \cdot 0$ with sodium hydroxide.

(3) M9 agar: dibasic sodium phosphate, 5.8 g.; monobasic potassium phosphate, 3.0 g.; ammonium chloride, 1.0 g.; sodium chloride, 0.5 g.; glucose, 2.0 g.; magnesium sulphate $\left(0 \cdot 7 \mathrm{H}_{2} \mathrm{O}\right), 250 \mathrm{mg}$.; calcium chloride $14 \mathrm{mg}$.; $1 \%$ gelatin solution, $10 \mathrm{ml}$; agar, 8.0 g. (Ionagar, Oxo Ltd., London).

(4) Peptone broth: Bacto-Peptone, 10.0 g. (Difco Laboratories, Detroit, Michigan, U.S.A.); Difco Beef Extract, 3.0 g.; glucose, 1.0 g.; sodium chloride, 5.0 g.

Phosphate-buffered saline was $1 \%$ sodium chloride in $0.02 \mathrm{M}$-phosphate buffer $(\mathrm{pH} \mathrm{6} \cdot 8)$.

Survival of u.v.-irradiated $T 1$ phage. The u.v. radiation source was a single $15 \mathrm{~W}$. General Electric germicidal lamp with a maximum output at $2537 \AA$. Calibrated with bacteriophage T2 according to the method of Latarjet, Morenne \& Berger (1953), this lamp delivered $15.4 \mathrm{ergs} / \mathrm{mm} .{ }^{2} / \mathrm{sec}$. at a distance of $51.5 \mathrm{~cm}$. from the source. T1 phage, $5 \times 10^{6}$ particles $/ \mathrm{ml}$, was irradiated with a dose of $616 \mathrm{ergs} / \mathrm{mm} .^{2}$ and $0.1 \mathrm{ml}$. was mixed with $2.5 \mathrm{ml}$. of warm $\left(48^{\circ}\right)$ semi-solid tryptone agar containing one drop of the overnight culture to be tested. The semi-solid mixtures were then overlayered on to fresh tryptone agar plates and the number of plaques counted after overnight incubation at $37^{\circ}$. This is essentially the rapid method described by Rörsch et al. (1962) for differentiating between mutants capable of 'reactivating' 
irradiated T1 phage and those unable to do so. When strains able to reactivate irradiated T1 phage were used as hosts, the plaques were confluent. When those unable to 'reactivate' irradiated T1 phage were used as hosts, a countable number of plaques were seen.

Survival of u.vi-irradiated bacteria. Cultures grown overnight in peptone broth were washed twice with buffered saline $(\mathrm{pH} \mathrm{6 \cdot 8)}$ and exposed with gentle agitation in $50 \mathrm{~mm}$. diameter Petri dishes containing $1 \mathrm{ml}$. of bacterial suspension $\left(2 \times 10^{6}\right.$ bacteria/ml.). Appropriate dilutions in cold phosphate-buffered saline were plated in duplicate on tryptone agar ( $\mathrm{pH} \mathrm{7 \cdot 0)}$ and on $\mathrm{M9}$ agar, incubated at $37^{\circ}$ for 24 and $48 \mathrm{hr}$. respectively, and counted. All manipulations subsequent to irradiation were done in subdued light to minimize photoreactivation. Sensitivity to u.v. radiation was calculated as the dose to give $10 \%$ survivors.

Measurement of resistance to chemical agents. Cultures grown overnight in peptone broth were adjusted with a model 9 Nepho-colorimeter (Coleman Instruments Inc.) to $3.5 \times 10^{8}$ bacteria $/ \mathrm{ml}$. and streaked on gradient plates according to the method of Szybalski \& Bryson (1952). Gradient plates were made with tryptone agar (pH 5.5) except that $\mathrm{pH} \mathbf{7 \cdot 8}$ was used in tests with proflavine; M9 agar was used in tests with azaserine. The minimum inhibitory concentration (M.I.C.) was determined as follows: (length of solid growth/total length of streak) $\times$ maximum concentration $(\mu \mathrm{g} . / \mathrm{ml}$.) of test compound.

\section{RESULTS}

Reactivation of u.v.-irradiated $T 1$ phage. With the following hosts the plaques

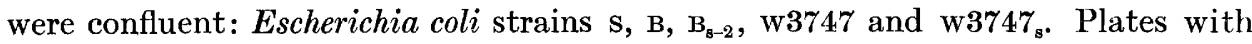
$E$. coli strain $B_{8-1}$ had 177 plaques; with strain $A B 1886,145$ plaques. To obtain numbers of plaques in this range for $E$. coli strains s, B, $B_{8-2}$, w3747 and $\mathrm{w}^{374} 7_{\mathrm{s}}$, the irradiated phage suspension had to be diluted $1 / 50$. When this was done there were no significant differences in the number of plaques produced by each strain in this group.

Response to u.v. radiation. Figures 1, 2 and 3 show the survival curves following ultraviolet irradiation of Escherichia coli strains $\mathbf{B}_{,} \mathbf{B}_{8-1}, \mathbf{B}_{\mathrm{g}-2}, \mathrm{~s}$, w3747, W3747, AB1157 and AB1886 when plated on tryptone agar and on M9 agar. The survival curves of the radioresistant parent strains AB1157 and w3747 (Fig. 3) indicate significant resistance, independent of plating medium, $860 \mathrm{ergs} / \mathrm{mm}^{2}{ }^{2}$ and 462 $\mathrm{ergs} / \mathrm{mm} .^{2}$ respectively, resulting in $10 \%$ survival.

Escherichia coli strains $\mathrm{B}_{\mathrm{B}-1}$ and $\mathrm{AB} 1886$ (Fig. 1) were similar in that: (1) they were significantly more sensitive to u.v. irradiation than the other strains; (2) their survival curves were exponential to more than three decades of kill; (3) both showed a limited plating-medium response: there were more survivors at any dose when the bacteria were plated on M9 medium than when plated on tryptone medium, but the survivors on M9 medium were considerably fewer than those of strains $B, B / r$, AB1157 or w3747. On the other hand, $E$. coli strains w3747, B, and s (Fig. 2) were related in that: (1) their survival curves were identical when plated on complete medium; (2) they exhibited a marked plating-medium response: strain w3747, could not be distinguished from its parent strain w3747 when both strains were plated on M9 medium after u.v. irradiation. 


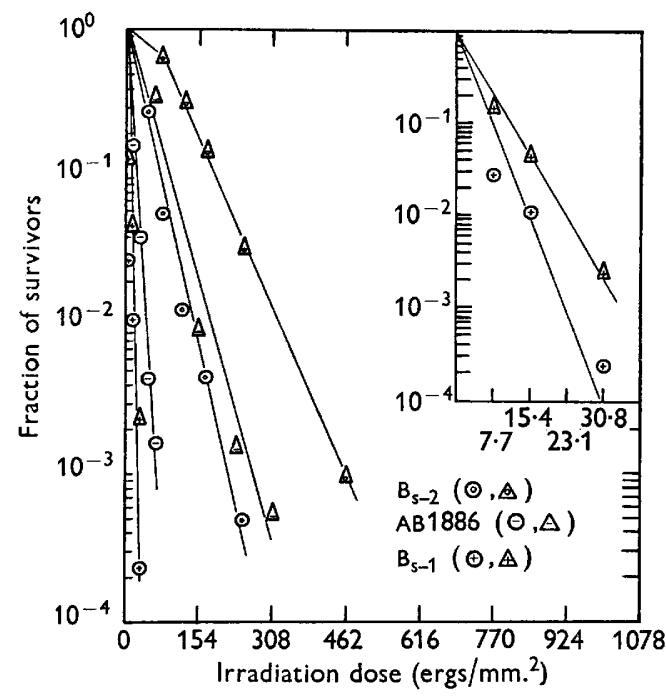

Fig. 1

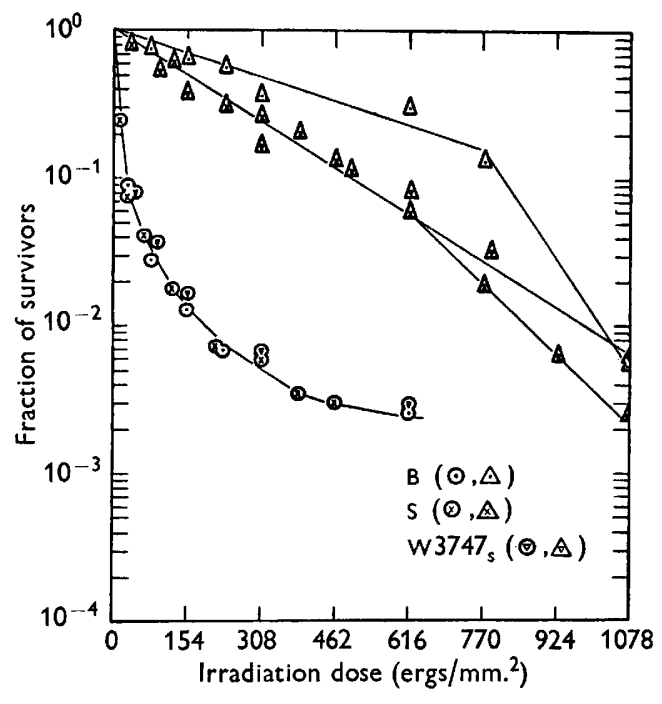

Fig. 2

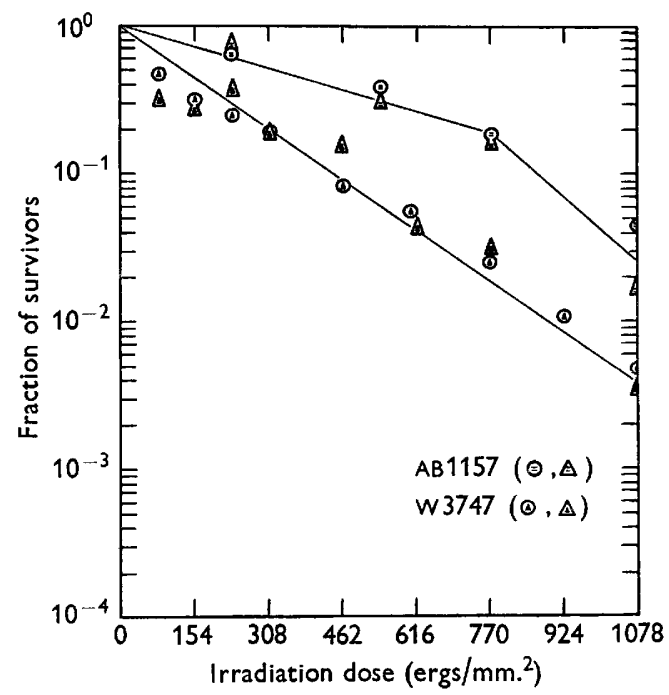

Fig. 3

Fig. 1. Survival of radiation-sensitive Escherichia coli strains $\mathbf{B}_{8-1}, \mathbf{B}_{8-2}$, and $\mathrm{k} 12$ AB1886 exposed to ultraviolet radiation and plated on tryptone agar, $\mathbf{p H ~ 7 . 0}(\oplus, \odot, \ominus)$ and м9 agar, $\mathrm{pH} 6.8(\mathbb{A}, \Delta, \Delta)$. Each point represents an average of 3 independent determinations for strains $B_{8-1}$ and $B_{8-2}$ and 6 for strain $A B 1886$. The insert in the upper righthand corner indicates the plating-medium response of strain $\mathbf{B}_{\mathrm{g}-1}$ on a more magnified scale.

Fig. 2. Survival of radiation-sensitive Escherichia coli strains $\mathrm{B}$, s, and $\mathrm{k12} \mathrm{war7}_{\mathrm{s}} \mathrm{ex}$ posed to ultraviolet radiation and plated on tryptone agar, $\mathrm{pH} \mathbf{7 \cdot 0}(\odot, \otimes, \otimes)$ and $\mathrm{m9}$ agar, $\mathrm{pH} 6 \cdot 8(\Delta, \Delta, \Delta)$. Each point represents an average of 5 independent determinations for each strain.

Fig. 3. Survival of radiation-resistant Escherichia coli strain $\mathrm{K} 12$ AB1157 and strain $\mathrm{K} 12$ w3747 exposed to ultraviolet radiation and plated on tryptone agar, $\mathrm{pH} 7 \cdot 0(\ominus,($ ) $)$ and M9 agar, pH $6.8(\Delta, \Delta)$. Each point represents an average of 2 to 4 independent determinations. 
The survival curve for Escherichia coli strain $\mathbf{B}_{8-2}$ (Fig. 1) was exponential for more than three decades of kill, but it showed greater sensitivity than strain B only at doses higher than 154 ergs/mm. ${ }^{2}$ (The survival curves for strains $\mathrm{B}_{\mathrm{B}-2}$ and $\mathrm{B}$ varied somewhat from those reported for these strains by Hill \& Simson in 1961, perhaps due to a difference in experimental procedures or to a genetic drift in the bacterial populations; Greenberg \& Woody-Karrer, in press.) Strain $\mathbf{B}_{8-2}$, like strain $\mathbf{B}_{\mathrm{g}-1}$, exhibited a limited plating-medium recovery when plated on M9 medium.

Table 2. Cross-resistance patterns of radiation-sensitive and radiation-resistant mutants of Escherichia coli

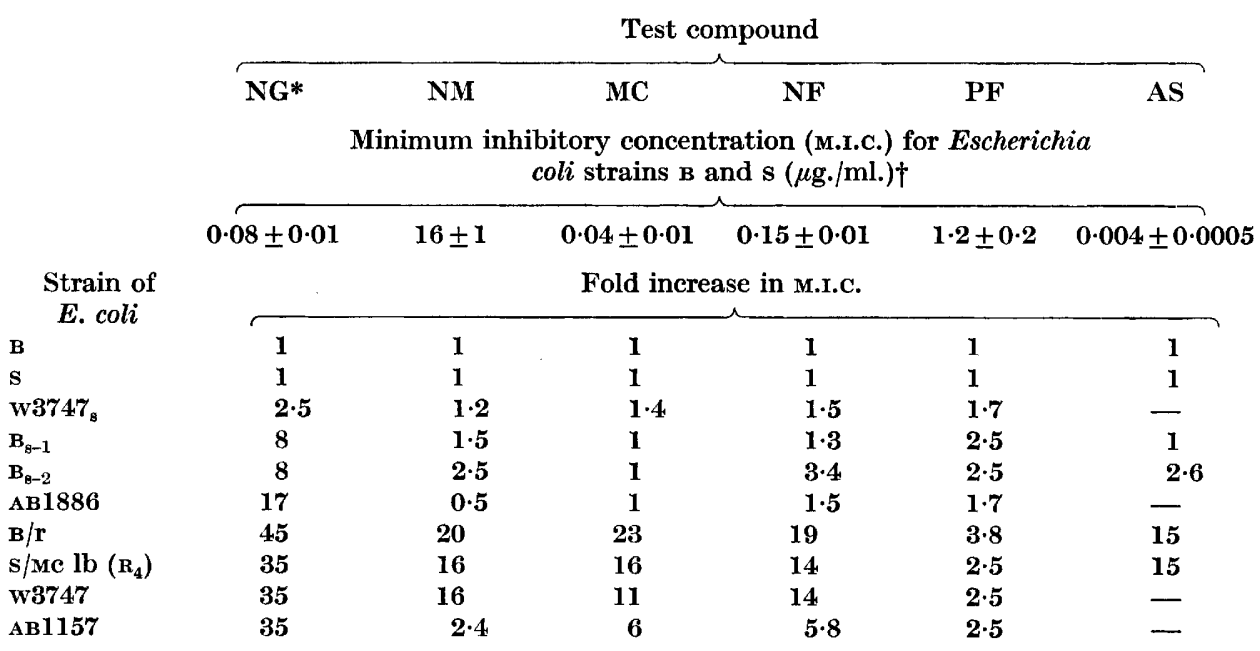

* The following abbreviations will be used: 1-methyl-3-nitro-1-nitrosoguanidine, NG; nitrogen mustard, NM; mitomycin C, MC; nitrofurazone (5-nitro-2-furaldehyde semicarbazone), NF; proflavine, PF; azaserine, AS.

$\dagger \mu \mathrm{g} . / \mathrm{ml}$. estimated from gradient plates (Szybalski \& Bryson, 1952).

Response to radiomimetic chemicals. Table 2 shows the minimum inhibitory concentrations of five radiomimetic chemicals for each of the strains studied relative to Escherichia coli strains B and s. The following points should be noted: (1) The crossresistance patterns of strains $\mathrm{w3747,} \mathrm{AB} 1157$, and $\mathrm{s} / \mathrm{Mc} \mathrm{lb}$, a prototype of the most frequently occurring radioresistant class of mutants $\left(\mathrm{R}_{4}\right)$ isolated from $\boldsymbol{E}$. coli strain $\mathrm{s}$, were similar to that of strain $\mathrm{B} / \mathrm{r}$. All four strains were very resistant to all the agents tested. (2) The cross-resistance pattern of strain $\mathrm{AB} 1886$, indicating sensitivity as compared with that of strain $\mathrm{AB1157}$, was very similar to those of strains $B_{8-1}$ and $B_{8-2}$, the most significant feature being resistance to nitrosoguanidine and proflavine. (3) The cross-resistance pattern of strain $\mathrm{w} 3747_{\mathrm{s}}$, indicating sensitivity as compared with that of strain w3747, most resembled those of $E$. coli strains $\mathrm{B}$ and $\mathrm{s}$, being relatively sensitive to all the radiomimetic agents tested. 


\section{DISCUSSION}

The evidence suggests that there are at least two different kinds of radiosensitive strains of Escherichia coli strain $\mathrm{K} 12$, one of which, strain $\mathrm{w}^{3} 747_{\mathrm{g}}$, is analogous to $E$. coli strains $\mathrm{B}$ and $\mathrm{s}$, the other, strain $\mathrm{AB} 1886$, analogous to $E$. coli strain $\mathrm{B}_{\mathrm{8}-\mathrm{I}}$ (and probably to strain $\mathbf{B}_{I I I}$, Rörsch et al. 1962). The u.v. survival curve of strain w3747 is identical with those of $E$. coli strains B and s when plated on tryptone medium, and all three strains exhibited a similar plating-medium response. Strains $\mathrm{w} 3747_{\mathrm{s}}, \mathrm{B}$ and $\mathrm{s}$ were similar in sensitivity to the five radiomimetic agents tested, whereas strain w3747 was about as resistant to these compounds as the $B / r$ and $R_{4}$ mutants of strain s. Finally, all three strains exhibited no measurable loss of ability to 'reactivate' irradiated T1 phage.

Escherichia coli strain $\mathrm{AB} 1886$, on the other hand, is very similar in many of its properties to $E$. coli strain $\mathbf{B}_{8-1}$. Following u.v. radiation, the survival curves of strain $\mathrm{AB} 1886$ are similar to those of strain $B_{8-1}$ in general shape, sensitivity, and plating-medium recovery. Strain $\mathrm{AB} 1886$ was sensitive, as compared with its radioresistant parent, to all of the radiomimetic agents studied, but was markedly less sensitive to nitrosoguanidine and somewhat less sensitive to proflavine than

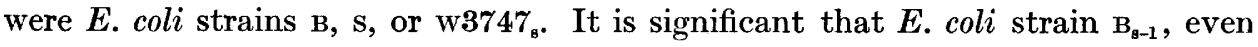
though more sensitive to u.v. radiation than its parent, strain $\mathbf{B}$, was no more sensitive than strain $\mathrm{B}$ to most of the radiomimetic agents tested and was significantly more resistant to nitrosoguanidine and proflavine than $\boldsymbol{E}$. coli strain B. Finally strains $B_{8-1}$ and $A B 1886$, in corroboration of reports by Ellison, Feiner \& Hill (1960) and Howard-Flanders \& Theriot (1962), did not reactivate U.v.-damaged T1 phage.

The locus which determines radiosensitivity in Escherichia coli strain $\mathrm{B}_{8-1}$ has not been mapped. However, Howard-Flanders et al. (1962) showed that the radiosensitivity of $E$. coli strain $\mathrm{AB} 1886$ is the result of a mutation $\left(U V^{\mathrm{R}}\right.$ to $U V^{\mathrm{s}}$ ) at a locus 0.37 the distance between the loci for arginine synthesis and arabinose fermentation; $U V^{\mathrm{R}}$ controls a mechanism, absent from $\boldsymbol{U} V^{\mathrm{s}}$ mutants, which reactivates damage done by ultraviolet radiation.

The radiosensitive mutant strain $\mathbf{B}_{\mathrm{III}}$, of Escherichia coli strain $\mathrm{B}$, isolated by Rörsch et al. (1962), is similar to $E$. coli strains $\mathrm{B}_{\mathrm{8}-\mathrm{I}}$ and $\mathrm{AB} 1886$ in its sensitivity to u.v. radiation and in its inability to reactivate $u . v$. -irradiated $\mathrm{T} 1$ phage. These investigators have shown that the radiosensitivity of mutant strain $\mathrm{B}_{\mathrm{III}}$ is the result of a mutation $\left(s y n^{+}\right.$to $\left.s y n^{-}\right)$at a locus between xylose fermentation and streptomycin resistance and closely linked to the former (Rörsch, Edelman \& Cohen, 1963). The syn and $U V$ loci, while on the same quadrant of the circular chromosome of $\boldsymbol{E}$. coli as depicted by Jacob \& Wollman (1961), do not map at the same place. It is possible that mutations at either of two different loci result in similar phenotypes, or that the linear arrangements of genes is not identical in at least this segment of the chromosome of $\boldsymbol{E}$. coli strains B and k12.

Cook \& Greenberg (unpublished observations) have demonstrated in crosses between radioresistant Escherichia coli strain $\mathrm{K} 12$ ( $\mathrm{Hfr} 1$ and 2) and E. coli strain $\mathrm{s}$ that there is a locus (which will be referred to as $R A$ to distinguish it from $U V$ ) for radioresistance closely linked to the loci for lactose fermentation and resistance to phage T6. The nature of the sensitivity $\left(R A^{\mathrm{s}}\right)$ or resistance $\left(R A^{\mathrm{R}}\right)$ conferred by the gene at this locus is under further investigation. The evidence as yet unpublished 
suggests that the sensitivity of strain $\mathrm{w} 3747_{\mathrm{g}}$ is a result of a mutation at or near this locus. Its parent strain w3747 can transmit radiation resistance along with the ability to ferment lactose by F-duction to E. coli strain s1 $\left(\mathrm{F}^{-} \mathrm{Lac}^{-}\right)$. Escherichia coli strain $\mathrm{K} 12 \mathrm{~W} 3 \mathbf{3 7 4} 7_{\mathrm{s}}$, while able to transmit together and at a high frequency its $\mathrm{Lac}^{+}$and $\mathrm{F}^{+}$characteristics, does not transmit radiation resistance to strain $\mathrm{sl}$. However, the $\mathrm{F}^{\prime}$ episome of strains w3747 and w3747, is extremely unstable in $E$. coli strain $\mathrm{s}$, rendering quantitative analysis difficult. A more promising approach to the genetics of the $R A$ locus would appear to be to 'cure' strain w3747, of its episome, making it available as an $\mathrm{F}^{-}$strain for recombination studies with donor strain K12s.

The fact that when Escherichia coli strain $\mathrm{k} 12$ becomes radiosensitive either by a mutation at the $\boldsymbol{U} \boldsymbol{V}$ or the $\boldsymbol{R A}$ locus it also becomes sensitive to Mitomycin $\mathrm{C}$, nitrogen mustard and nitrofurazone, can be interpreted to mean that some of the damage to the cell caused by these agents is similar to that caused by u.v. radiation. If the $U V$ locus controls a repair mechanism this same mechanism might also repair damage done by radiomimetic chemicals. Howard-Flanders et al. (1962) suggested that $U V^{\mathrm{R}}$ reactivation acts on certain photoproducts associated with thymine in DNA. There is as yet no evidence that radiomimetic agents produce any changes in DNA thymine comparable to the thymine dimerization produced by u.v. radiation (Beukers \& Berends, 1961). Nor is there any evidence that the damage done by radiomimetic agents can be photoreactivated as can thymine dimers (Setlow \& Setlow, 1961; Wulff \& Rupert, 1962). It is possible that the $U V^{\mathrm{R}}$ mechanism repairs other kinds of damage to DNA as well as thymine photoproducts. It is clear that the $U V^{\mathrm{R}}$ mechanism does not significantly repair damage done by nitrosoguanidine or proflavine since $U V^{\text {s }}$ organisms are relatively resistant to these chemicals. In fact, in the mutation from $E$. coli strain B to strain $\mathrm{B}_{\mathrm{g}-1}$, in which sensitivity to ultraviolet radiation increases and sensitivity to Mitomycin $\mathrm{C}$ remains essentially unchanged, sensitivity to nitrosoguanidine and proflavine decreases. This is difficult to reconcile with the observation that in radioresistant mutants of $E$. coli strain $\mathbf{B}$, resistance to all the radiomimetic agents increases along with increased resistance to radiation, both u.v. and X-ray (Greenberg \& Woody-Karrer, 1963). It is also difficult at present to classify $E$. coli strain $B_{8-2}$, which resembles $E$. coli strain $\mathbf{B}_{\mathrm{B}-1}$ in all its properties including a decreased sensitivity to nitrosoguanidine and proflavine, but is still able to reactivate $u . v$. -damaged phage. Strain $\mathbf{B}_{\mathbf{s}-2}$ may represent another class of radiosensitive mutants.

The mechanisms responsible for the type of radiosensitivity observed in Escherichia coli strains $\mathrm{B}, \mathrm{s}$, and $\mathrm{w}^{3747_{\mathrm{s}}}$ may result from the lack or loss of an enzyme able to repair kinds of damage different from that repaired by the $U V$ locus. Apparently, for strain w3747 to exhibit the degree of radioresistance observed the $U V$ and $R A$ loci must both be functional. It is not yet clear what the genetic changes are which account for radiation resistance in the various radioresistant mutants of $\boldsymbol{E}$. coli strains B and s (Greenberg \& Woody-Karrer, 1963). The fact that there are many different radioresistant mutants of strains $B$ and $s$ would suggest that either mutations in a number of different modifiers of the $R A$ locus or a variety of changes within the $\boldsymbol{R} \boldsymbol{A}$ locus result in different radioresistant phenotypes. 
This work was carried out under U.S. Public Health Service Grant No. CY-5687. The technical assistance of Miss C. R. Woody is gratefully acknowledged.

\section{REFERENCES}

Adler, H. I. \& Copeland, J. C. (1962). Genetic analysis of radiation response in Escherichia coli. Genetics, 47, 701 .

Alper, T. \& Grllies, N. E. (1960). The relationship between growth and survival after irradiation of Escherichia coli strain B and two resistant mutants. J. gen. Microbiol. 22, 113.

Beukers, R. \& Berends, W. (1961). The effects of UV irradiation on nucleic acids and their components. Biochim. biophys. Acta, 49, 181.

Bryson, V. (1948). The effects of nitrogen mustard on Escherichia coli. J. Bact. 56, 423.

Ellison, S. A., Feiner, R. \& Hill, R. F. (1960). A host effect on bacteriophage survival after ultraviolet irradiation. Virology, 11, 294.

Greenberg, J., Mandell, J. D. \& Woody, P. L. (1961a). Resistance and cross-resistance of Escherichia coli mutants to radiomimetic agents. A preliminary report. Cancer Chemotherapy Reports no. 11, p. 51.

Greenberg, J., Mandeld, J. D. \& Woody, P. L. (1961 b). Resistance and cross-resistance of Escherichia coli mutants to antitumour agent Mitomycin C. J. gen. Microbiol. 26, 509.

Greenberg, J. \& Woody-Karrer, P. (1963). Radioresistant mutants of Escherichia coli B (ORNL). Radiat. Res. (in the Press).

Hilt, R. F. (1958). A radiation-sensitive mutant of Escherichia coli. Biochim. biophys. Acta, 30, 636.

HilL, R. F. \& Simson, E. (1961). A study of radiosensitive and radioresistant mutants of Escherichia coli strain B. J. gen. Microbiol. 24, 1.

Hirota, Y. \& Sneath, P. H. A. (1961). $\mathbf{F}^{\prime}$ and F mediated transduction in Escherichia coli к 12. Jap. J. Genet. 36, 307.

Howard-Flanders, P. \& Theriot, L. (1962). A method for selecting radiation-sensitive mutants of Escherichia coli. Genetics, 47, 1219.

Howard-Flanders, P., Boyce, R. P., Simson, E. \& Theriot, L. (1962). A genetic locus in $E$. coli $\mathrm{K} 12$ that controls the reactivation of UV-photoproducts associated with thymine in DNA. Proc. nat. Acad. Sci., Wash. 48, 2109.

JacoB, F. \& Wollman, E. (1961). Sexuality and the Genetics of Bacteria. New York and London: Academic Press.

Latarjet, R., Morenne, P. \& Berger, R. (1953). Un appareil simple pour le dosage des rayonnements ultraviolets émis par les lampes germicides. Ann. Inst. Pasteur, 85,174 .

Mandell, J. D., Woody, P. L. \& Greenberg, J. (1961). Resistance and cross-resistance of Escherichia coli mutants to anticancer agents. 1-methyl-3-nitro-1-nitrosoguanidine. J. Bact. 81, 419.

Roberts, R. B. \& Aldous, E. (1949). Recovery from ultraviolet irradiation in Escherichia coli. J. Bact. 57, 363.

Rörsch, A., Edflman, A., van der Kamp, C. \& Cohen, J. A. (1962). Phenotypic and genotypic characterization of radiation sensitivity in Escherichia coli B. Biochim. biophys. Acta, 61, 278.

Rörsch, A., Edelman, A. \& Cohen, J. A. (1963). The gene-controlled radiation sensitivity in Escherichia coli. Biochim. biophys. Acta, 68, 263.

Setlow, J. \& Setlow, R. B. (1961). Ultraviolet action spectra of ordered and disordered DNA. Proc. nat. Acad. Sci., Wash. 47, 1619.

Szybalski, W. \& Bryson, V. (1952). Genetic studies on microbial cross-resistance to toxic agents. I. Cross-resistance of Escherichia coli to fifteen antibiotics. J. Bact. 64, 489.

Szybalski, W. \& Nelson, T. C. (1954). Genetics of bacterial resistance to nitrofurans and radiation. Bact. Proc. p. 51. 
Witkin, E (1947). Genetics of resistance to radiation in Escherichia coli. Genetics, 32, 221.

Woody, P. L., Mandell, J. D. \& Greenberg, J. (1961). Resistance and cross-resistance of Escherichia coli mutants to anticancer agents. Nitrogen mustard and nitromin. Radiat. Res. 15, 290.

Woody-KArRer, P. \& Greenberg, J. (1963). Resistance and cross-resistance of Escherichia coli $\mathrm{S}$ mutants to the radiomimetic agent nitrofurazone. J. Bact. 85, 1208.

WulfF, D. L. \& Rupert, C. S. (1962). Disappearance of thymine photodimer in ultraviolet irradiated DNA upon treatment with a photo-reactivating enzyme from baker's yeast. Biochem. biophys. Res. Comm. 7, 237. 